\title{
The kinetics of crossflow dynamic membrane bioreactor
}

\author{
Fang $\mathrm{Li}^{*}$, Jihua Chen and Chunhua Deng \\ College of Environmental Science and Engineering, Donghua University, Shanghai 200051, China
}

\begin{abstract}
Crossflow dynamic membrane bioreactor (CDMBR) kinetics was investigated by treating caprolactam wastewater over a period of $180 \mathrm{~d}$. The removal efficiencies of organic substances and nitrogen averaged over $99 \%$ and $80 \%$, respectively. The observed sludge yield was only $0.14 \mathrm{~g} \mathrm{SS} \cdot \mathrm{g}^{-1} \mathrm{COD} \cdot \mathrm{d}^{-1}$ at an SRT of $30 \mathrm{~d}$ and sludge organic loading about $0.3 \mathrm{~g} \mathrm{COD} \cdot \mathrm{g}^{-1}$ SS $\cdot \mathrm{d}^{-1}$. Based on total organic carbon TOC measurement, accumulation of soluble microbial products (SMP) in the supernatant caused by membrane separation was observed during the initial operational period. SMP in the supernatant was verified biodegradable subsequently through molecular weight distribution (MWD) analysis. Specific oxygen uptake rate (sOUR) decreased with MLSS increasing in the reactor, which could be expressed by a exponential function. sOUR maintained in the range of 4-5 $\mathrm{mg} \mathrm{O}_{2} \cdot \mathrm{h}^{-1} \cdot \mathrm{g}^{-1}$ VSS while oxygen uptake rate (OUR) increased with MLSS concentration. Despite the low sOUR level of Nitrosomo and Nitrobacter, nitrifying capacity of CDMBR remained high enough to qualify nitrogen removal.
\end{abstract}

Keywords: CDMBR, caprolactam wastewater, nitrogen removal, sOUR

\section{Introduction}

The membrane bioreactor (MBR) has proved to be an attractive process for wastewater treatment with considerable advantages over conventional treatment methods (Arnot and Zahir, 1996; Gander et al., 2000; Roberts et al., 2000). However, some problems on bio-fouling and cake formation limit the acceptance of MBR in wastewater treatment, especially for crossflow membrane bioreactors (CMBR). CMBR is challenged by the submerged membrane bioreactor (SMBR) because of its intrinsic weaknesses, such as rapid flux decrease and low viability of biomass. Brockma and Seyfried (1996) believed that high intensity of shear stress induced by the pump significantly influenced the activity of sludge in CMBR. Soluble microbial product which would accumulate in the reactor by membrane rejection could inhibit the micro-organisms, especially the nitrifying bacteria, reported by Chudoba (1985), Rappaport et al. (1979), Ross et al. (1998). In addition, Kim et al. (2001) reported that extracellular polymeric substances (EPS) were readily liberated from flocs due to the centrifugal pump shear stress, which led to an increase in SMP concentration.

Cake formation is unavoidable in crossflow filtration. However, this phenomenon can be exploited innovatively by forming a purpose-built dynamic membrane (DM) to substitute the involuntary layer. It was reported that pre-coated DM could reduce the resistance of irreversible fouling and simplify the cleaning operation in wastewater treatment (Megat and Fakhrul, 2002; Muhammad and Anderson, 1997; Marc, et al., 1999).The satisfactory performance of pre-coated DM in activated sludge filtration was also verified in a bench-scale experiment ( $\mathrm{Li}$ et al., 2005). No information is available yet on the combination of pre-coated DM and CMBR.

The purpose of this study was to investigate the kinetics of improved CMBR combined with pre-coated DM. The quality of

\footnotetext{
* To whom all correspondence should be addressed.

证 +8621 62373614; fax: +8621 62193096;

e-mail: lifang@mail.dhu.edu.cn

Received 17 May 2005; accepted in revised form 31 December 2005.
}

permeate and supernatant, as well as sOUR, was monitored in order to evaluated the performance of DM on SMP rejection and the influence of SMP on micro-organisms.

\section{Materials and methods}

\section{Caprolactam wastewater}

Caprolactam $\left(\mathrm{C}_{6} \mathrm{H}_{11} \mathrm{NO}\right)$ textile wastewater is difficult to treat due to its high nitrogen concentration. Table 1 gives the principal characteristics of the synthetic caprolactam wastewater adopted in this investigation.

\begin{tabular}{|c|c|c|c|c|c|}
\hline \multicolumn{7}{|c|}{ TABLE 1 } \\
\hline Items & $\begin{array}{c}\mathbf{C O D} \\
\left(\mathbf{m g} \cdot \boldsymbol{\ell}^{-1}\right)\end{array}$ & $\begin{array}{c}\mathbf{B O D}_{\mathbf{5}} \\
\left(\mathbf{m g} \cdot \boldsymbol{\ell}^{-1}\right)\end{array}$ & $\mathbf{p H}$ & $\begin{array}{c}\mathrm{TP} \\
\left(\mathbf{m g} \cdot \mathbf{\ell}^{-1}\right)\end{array}$ & $\begin{array}{c}\mathrm{TN} \\
\left(\mathbf{m g} \cdot \mathbf{\ell}^{-1}\right)\end{array}$ \\
\hline Conc. & 1800 & 1370 & 6.8 & 20 & 100 \\
& -2000 & -1530 & -7.2 & -30 & -130 \\
\hline
\end{tabular}

\section{Experimental system and operating conditions}

The schematic diagram of the crossflow dynamic membrane bioreactor used in this experiment is shown in Fig. 1. Caprolactam wastewater was pumped to the anoxic reactor (effective volume $=8 \ell$ ) fitted with a magnetic stirrer. The mixed liquor was fed gravimetrically to the aerobic reactor (effective volume $=20 \ell)$ and recirculated to the anoxic reactor for denitrification. The ratio of recirculation over influent flow rate was maintained at 3:1 throughout this experiment. The temperature was controlled at $30^{\circ} \mathrm{C}$ with a heat exchanger and dissolved oxygen (DO) concentration of over $3.0 \mathrm{mg} \cdot \ell^{-1}$ in the aerobic reactor. The trans-membrane pressure was provided by centrifugal pump. Both the retentate and the excess permeate were returned to the aerobic reactor to keep the reactor volume constant. No excess sludge was discharged until MLSS had been increased to $4500 \mathrm{mg} \cdot \ell^{-1}$ and then the sludge retention time (SRT) was controlled for $30 \mathrm{~d}$. Hydraulic retention time was 33 to $66 \mathrm{~h}$ to maintain the average sludge loading about $0.3 \mathrm{~g} \mathrm{COD} \cdot \mathrm{g}^{-1} \mathrm{SS} \cdot \mathrm{d}^{-1}$. During the experiment, the trans-mem- 


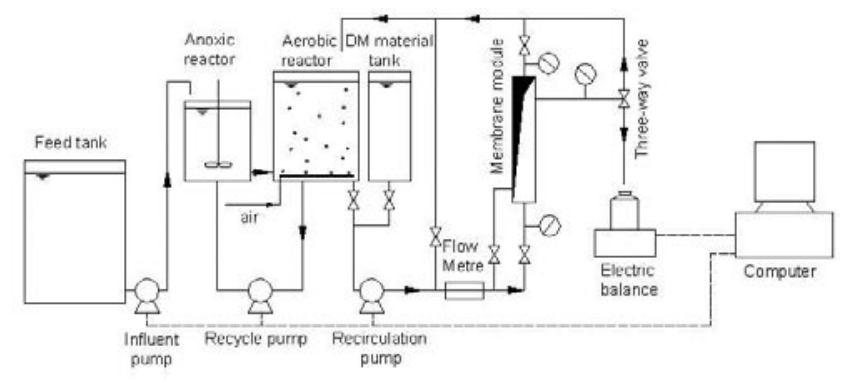

Figure 1

Schematic diagram of the experiment system

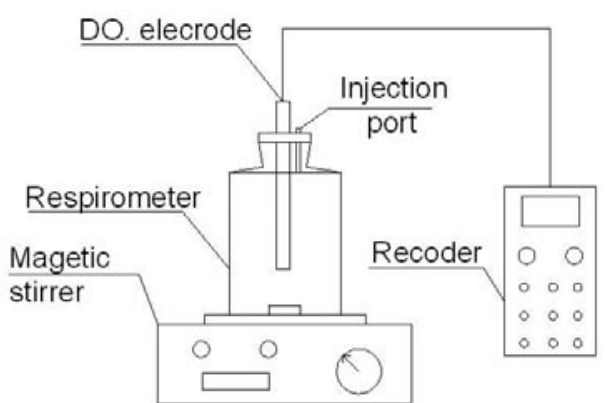

Figure 2

Batch respirometer used for recording OUR profile

brane pressure adopted was in the range of 0.1 to $0.2 \mathrm{MPa}$ and crossflow velocity in the range of 1 to $2 \mathrm{~m} \cdot \mathrm{s}^{-1}$.

This membrane was obtained from Futao Co. (Guangdong, China) and was a tubular ceramic membrane with an average surface pore size of $2 \mu \mathrm{m}$. The membrane module was made up of a stainless steel cylinder equipped with six tubes (each tube with an internal diameter of $9 \mathrm{~mm}$ and a length of $400 \mathrm{~mm}$ ) inside with a total membrane surface $0.0672 \mathrm{~m}^{2}$.

\section{DM preparation}

DM was formed on the tubular ceramic support by circulating the kaolinite solution of $0.5 \mathrm{~g} \cdot \ell^{-1}$ through the membrane module with the permeate outlet valve open under the designed condition, i.e. crossflow velocity of $1.0 \mathrm{~m} \cdot \mathrm{s}^{-1}$ and trans-membrane pressure of $0.2 \mathrm{MPa}$. The average particle size of kaolinite was $2 \mu \mathrm{m}$. Routine cleaning was carried out by hydraulic backpressure washing as the flux decreases less than $20 \ell \cdot \mathrm{m}^{-2} \cdot \mathrm{h}^{-1}$.

\section{Analytical methods}

The performance of the CDMBR was monitored by analysing influent, effluent and supernatant in aerobic reactor samples for selected parameter according to standard procedures, including $\mathrm{COD}_{\mathrm{Cr}}, \mathrm{BOD}_{5}, \mathrm{TN}, \mathrm{NH}_{4}^{+}-\mathrm{N}, \mathrm{NO}_{3}^{-}-\mathrm{N}, \mathrm{NO}_{2}^{-}-\mathrm{N}$ and $\mathrm{pH}$ (Chinese SEPA, 1997). The supernatant sample was obtained by centrifuging the mixed liquid at $6000 \mathrm{r} \cdot \mathrm{min}^{-1}$ for $10 \mathrm{~min}$, followed by filtration through a membrane of $0.45 \mu \mathrm{m}$. DO was measured by Oxi-330 Oximeter.

OUR has been a common method for measuring sludge activity rates because the most remarkable phenomenon is the depletion of DO in micro-organism metabolism. In order to distinguish the OUR values of different bacteria, allythiourea (ATU) and $\mathrm{NaClO}_{3}$ were selected as nitrification inhibitors as ATU is a selective inhibitor of the Nitrosomonas group and $\mathrm{NaClO}_{3}$ of the Nitrobacter group. A conventional batch feed respirometer with

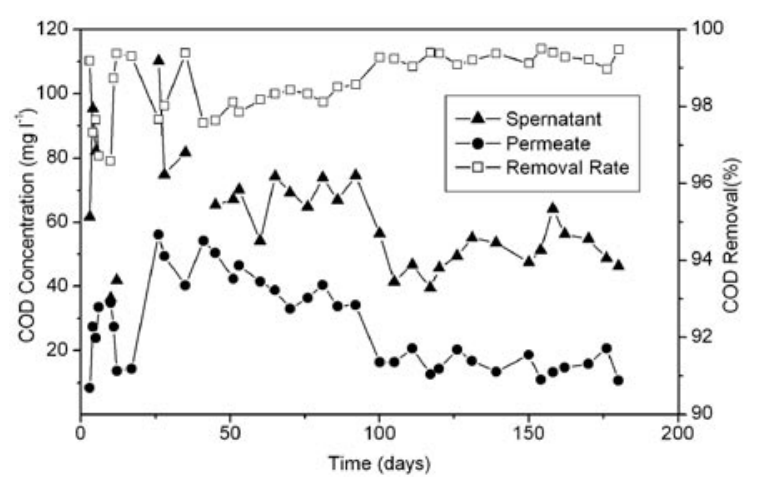

Figure 3

$C O D_{C r}$ concentration in supernatant and permeate of $C D M B R$

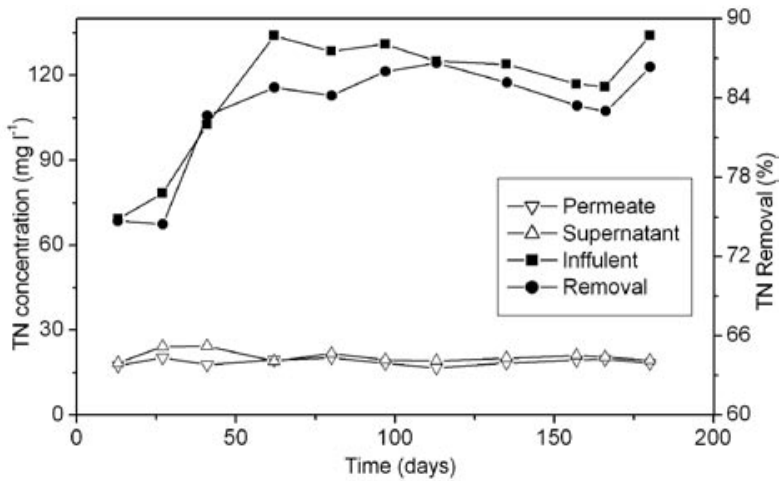

Figure 4

$T N$ concentration in supernatant and permeate of $C D M B R$

a small, completely closed reactor vessel (volume $=200 \mathrm{ml}$ ) was used for the experiment, as shown in Fig. 2 (Nowak and Svardal, 1997; Gorska, 1996).

\section{Results and discussion}

\section{Overall performance of the CDMBR}

$\mathrm{COD}_{\mathrm{Cr}}$ removal in the CDMBR is illustrated in Figs. 3 and 4. $\mathrm{COD}_{\mathrm{Cr}}$ concentration in the supernatant represented the removal efficiency in the bioreactors, while that in membrane permeate depicted the removal efficiency in the total CDMBR processes. During the operational period, the concentration of $\mathrm{COD}_{\mathrm{Cr}}$ in the supernatant was kept below $100 \mathrm{mg} \cdot \ell^{-1}$ while that of the permeate was below $50 \mathrm{mg} \cdot \ell^{-1} \mathrm{~d}$, even less than $20 \mathrm{mg} \cdot \ell^{-1}$ after $100 \mathrm{~d}$ operation, which illustrated that excellent membrane separation was achieved.

The TN removal and nitrification efficiency are shown in Figs. 4 and 5. The removal efficiency of TN was more than $80 \%$ and the concentration of TN in the membrane permeate was less than $20 \mathrm{mg} \cdot \ell^{-1}$. The difference in the TN concentration between membrane permeate and supernatant liquid was not obvious, which meant that there was no contribution of membrane to nitrogen removal. The $\mathrm{NH}_{4}{ }^{+}-\mathrm{N}$ concentration maintained at a low level, fluctuating in the range of 1 to $3 \mathrm{mg} \cdot \ell^{-1}$, as shown in Fig. 5, while nitrate and nitrite concentration remained at a high level. It can be explained that nitrification in the aerobic reactor was effective while denitrification in anoxic reactor was a restricting factor for nitrogen removal. The carbon source was supposed to be inadequate for denitrification in the anoxic tank. However, during the operational period, the treatment efficiency was satisfactory. 


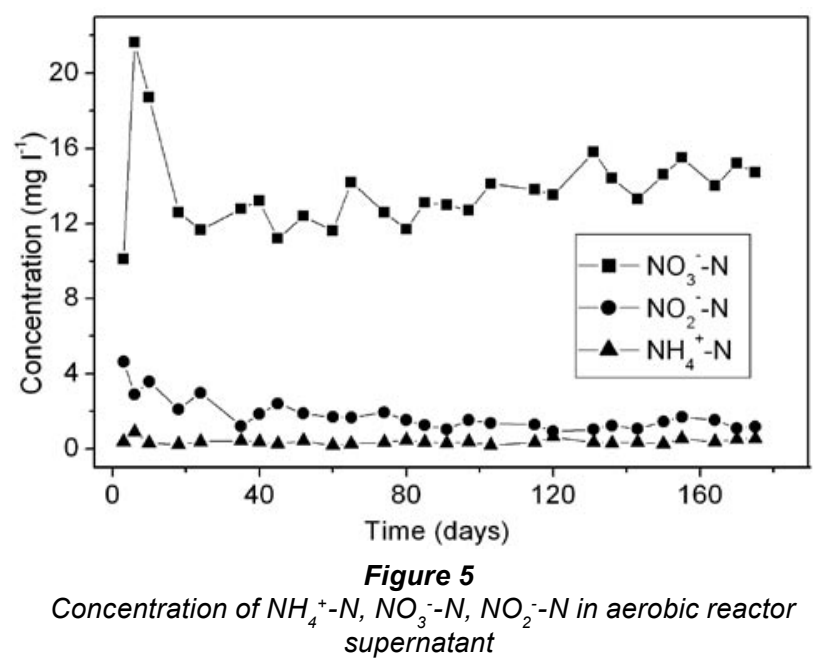

As for variations of MLSS and MLVSS, as shown in Fig. 6, both MLSS and MLVSS concentration decreased rapidly at first, e.g. MLSS decreasing from $3258 \mathrm{mg} \cdot \ell^{-1}$ to $1512 \mathrm{mg} \cdot \ell^{-1}$. During this period, no sludge was discharged from the reactor. $\mathrm{CHOO}$ and LEE (1996) found a similar phenomenon on anaerobic MBR and considered that the microbial cells moved from the bioreactor to the membrane surface. It can also be assumed that the sludge flocs were broken into smaller debris by shear stress from the pump, which was in favour of oxygen transfer for microorganisms to enhance endogenous respiration. After the initial stage, an increase in MLSS was observed. From day 45 onwards, excess sludge discharge was observed and the SRT was maintained at about $30 \mathrm{~d}$. The MLSS concentration increased until it reached $9213 \mathrm{mg} \cdot \ell^{-1}$ and then remained constant at this value. The observed sludge yield which was much lower than that of the conventional sludge process was established to be $0.14 \mathrm{~g}$ $\mathrm{SS} \cdot \mathrm{g}^{-1} \mathrm{COD} \cdot \mathrm{d}^{-1}$ by means of Eq. (1):

$$
Y=\Delta S S / \triangle C O D
$$

where:

$Y$ is the observed sludge yield

$\triangle S S$ is the amount of excessive sludge per day

$\triangle C O D$ is the amount of substrate utilised per day

The ratio of VSS/SS tended to increase in the initial stages and then levelled off ranging from $85 \%$ to $90 \%$ after excess sludge discharging. It was assumed that dissipation of some kaolin particles of the dynamic membrane into the mixed liquor led to the ratio of VSS/SS being kept low.

\section{Contribution of DM rejection}

Acknowledging the presence of SMP has revolutionised the understanding of wastewater treatment systems. In this study, the assumption is that caprolactam was completely removed in the reactor. In that event, the amount of SMP in MBR can be simplified to TOC in the reactor supernatant (Shin and Kang, 2003).

TOC concentration in the supernatant increased gradually up to $60 \mathrm{mg} \cdot \ell^{-1}$ during the first stage (day 1 to 35 ), then decreased to $24 \mathrm{mg} \cdot \ell^{-1}$ (day 100), as shown in Fig. 7. In the later stage of day 80 to day 180 , TOC became stable at near $18 \mathrm{mg} \cdot \ell^{-1}$. As for membrane permeate, concentration of TOC fluctuated very little. This implies that most of the SMP was retained in the reactor by membrane separation. The average pore size of the dynamic

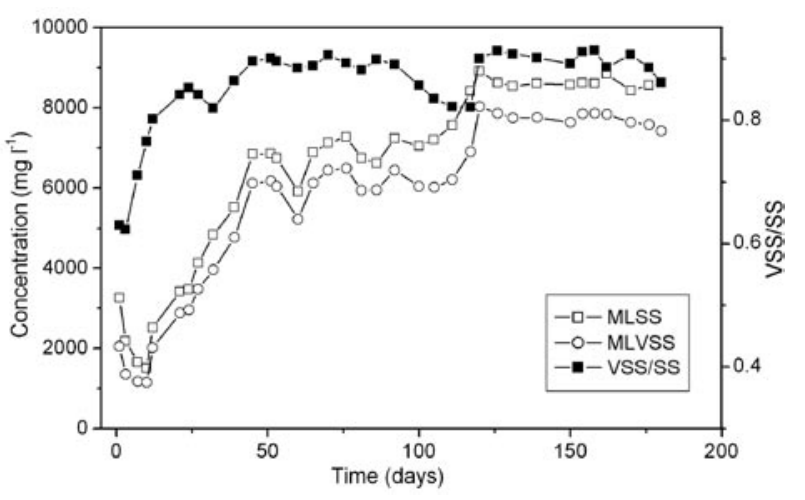

Figure 6

Variation of MLSS and MLVSS in the reactor

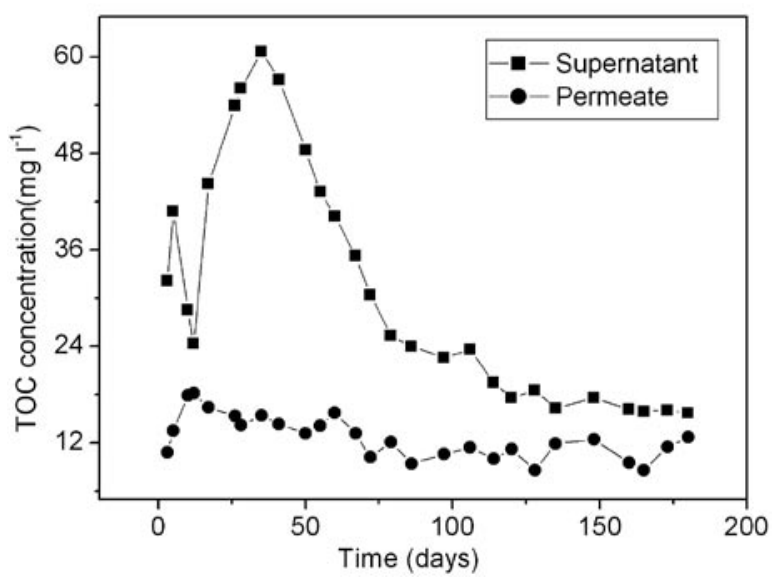

Figure 7

Variation of TOC of the supernatant and permeate

membrane surface was about $0.4 \mu \mathrm{m}$ which could not cut off most of the SMP. Due to pore blockage and cake formation, the membrane pores were gradually becoming narrower. The higher MW organic substrate was retained by the dynamic membrane and accumulated in the supernatant by membrane separation. However, according to the TOC trend in the supernatant shown in Fig. 7, a fraction of SMP could still be degraded biologically although it required quite a long acclimation period. As the operational time increased, the SMP level was kept constant because of the biological degradation and sludge withdrawal. Similar results were reported by other investigators (Shin and Kang, 2003; Huang X, et al., 2000).

In order to investigate the SMP transformation, the MWD was examined, as shown in Fig 8. Low MW compounds with an MW of lower than 10000 Da accounted for $72 \%$ at Day 5 . After $76 \mathrm{~d}$, the percentage of high MW compounds increased obviously and those of over 50000 Da increased to $36 \%$. On Day 135, the organic substrate with an MW in the range of $10000 \sim 50000$ shifted to $48 \%$ while those with an MW of more than $50000 \mathrm{Da}$ increased to $24.7 \%$. It implied that the high MW compounds, i.e. accumulated SMP, were broken down into smaller ones during the long retention time. This has also been verified by other investigators (Duncan and David C, 1999; Rittmann et al., 1987).

\section{Variation of sludge activity}

To verify the effect on biomass activity induced by the pump shear stress and accumulated SMP, OUR was measured. 


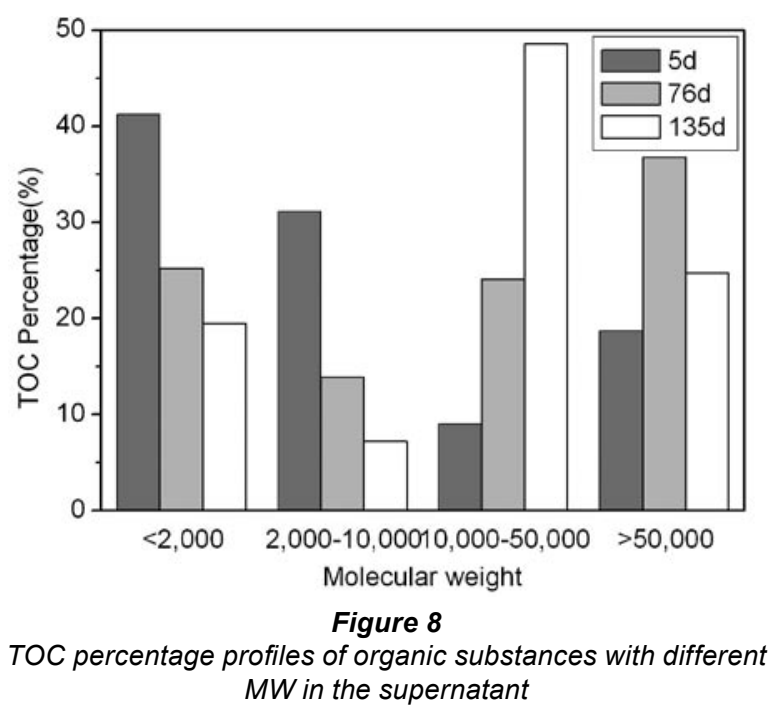

The sOUR, a very informative parameter on the biomass activity, was calculated as follows:

$s O U R=$ OUR/MLVSS

As shown in Fig. 9, the values of OUR and sOUR collapsed as the CDMBR system commenced. It can be explained that the sludge flocs were broken down by the pump shear stress and a large proportion of the micro-organism population mass perished as a result. A recovery was observed on Day 5. In the following operation, there was a tendency for OUR to increase and for sOUR to decrease respectively. The sOUR was maintained in the range of 4 to $5 \mathrm{mg} \mathrm{O} \cdot \mathrm{h}^{-1} \cdot \mathrm{g}^{-1}$ VSS and OUR increased with MLSS concentration after 50 days' operation. Compared with other investigators' reports, the value of sOUR in this study was lower. The values of sOUR, according to Halii's report, were much higher than those in this study under various sludge concentrations in submerged MBR (Halil et al, 2002). Brockmann reported that sOUR in CMBR was $5.2 \mathrm{mg} \mathrm{O}_{2} \cdot \mathrm{h}^{-1} \cdot \mathrm{g} \mathrm{VSS}^{-1}$. As suspected, pump shear stress and accumulated SMP should be responsible for low sOUR in CDMBR. Additionally, comparatively lower sludge organic loading possibly induced this phenomenon.

From the relationship between sOUR and MLSS, as shown in Fig. 10, it was noted that sOUR decreased with increased MLSS. On day 12 , sOUR was $3.4 \mathrm{mg} \mathrm{O} \cdot \mathrm{h}^{-1} \cdot \mathrm{g}^{-1} \mathrm{SS}$ with an MLSS of $2821 \mathrm{mg} \cdot \ell^{-1}$, while it was only $1.3 \mathrm{mg} \mathrm{O} \cdot \mathrm{h}^{-1} \cdot \mathrm{g} \mathrm{SS}^{-1}$ with an MLSS of $7220 \mathrm{~g} \cdot \ell^{-1}$ on Day 39. The relationship can be described as follows:

$$
\text { SOUR }=3.927 \exp (-1.487 \text { MLSS })
$$

In general, the total bacterial activity was directly proportional to bacterial numbers. However, high bacterial density would cause a decline of the unit organisms' activity. Additionally, bacterial activity showed a degree of cell metabolisability and enzymolysis reaction. It also related to the concentration of substrate. An increase in wastewater strength leads to an increase in micro-organism metabolic activity and higher OUR. F/M ratio would decline with the increasing of MLSS as long as the influent strength did not change. Because of the high MLSS, the sludge loading was below $0.3 \mathrm{~g} \mathrm{COD} \cdot \mathrm{g}^{-1} \mathrm{SS} \cdot \mathrm{d}^{-1}$ in the late operational period.

The sOUR tendency of heterotrophic organisms, Nitrosomonas group and Nitrobacter group is shown in Fig. 10. The collapse of the sOUR during the start-up period was due to the

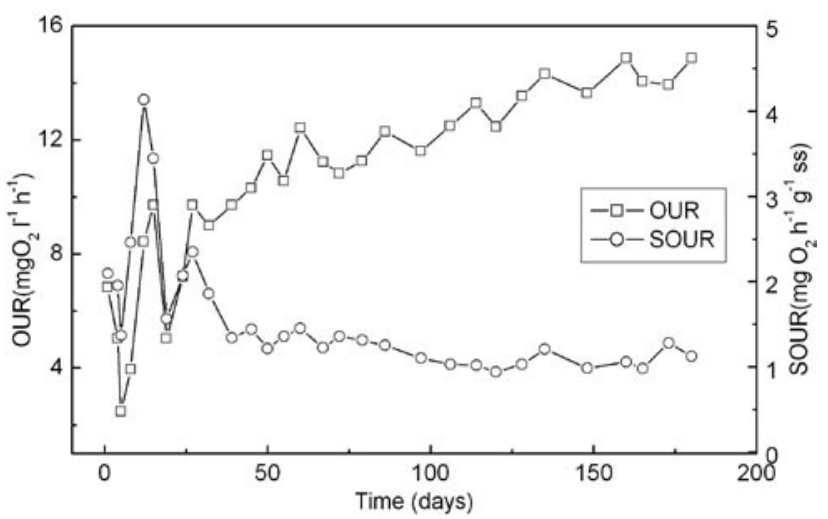

Figure 9

Variation of OUR and sOUR over the operation time

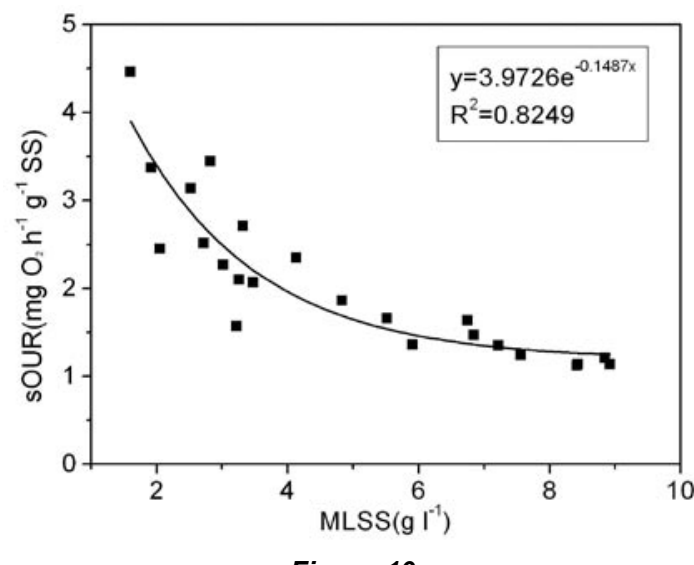

Figure 10

The relationship between sOUR and MLSS

hydraulic impact. Such phenomenon was observed by Wouter et al. (1999). sOUR of these micro-organisms performed a wave crest on Day 12 and levelled off afterwards. When comparing Fig. 7 and Fig. 11, it is clear that accumulation of SMP influenced the activities of the Nitrosomonas group and the Nitrobacter group. When the TOC concentration in the supernatant achieved a certain level, activities of the Nitrosomonas group and the Nitrobacter group began to decrease. After day 50 , both of these groups were stable at 0.11 and 0.24 $\mathrm{mg} \mathrm{O} \cdot \ell^{-1} \cdot \mathrm{g}^{-1}$ VSS. It was supposed that SMP would inhibit these micro-organisms finitely. Thus, these micro-organisms could adapt the bulk condition after an operational period. Even if a decrease of the specific sludge activity occurred, the nitrifying capacity still remained high enough to complete nitrification, which could be verified through supernatant quality.

\section{Conclusion}

- Satisfactory organic and nitrogen removal in CDMBR could be achieved in the treatment of a synthetic caprolactam wastewater. COD and nitrogen removal efficiencies were up to $99 \%$ and $80 \%$, respectively. The observed sludge yield was only $0.14 \mathrm{~g} \mathrm{SS} \cdot \mathrm{g}^{-1} \mathrm{COD} \cdot \mathrm{d}^{-1}$ at an SRT of $30 \mathrm{~d}$.

- Accumulation of supernatant SMP in the reactor by DM separation was observed during the initial operational period; subsequently it was verified to be biodegradable according to MWD in the supernatant. 


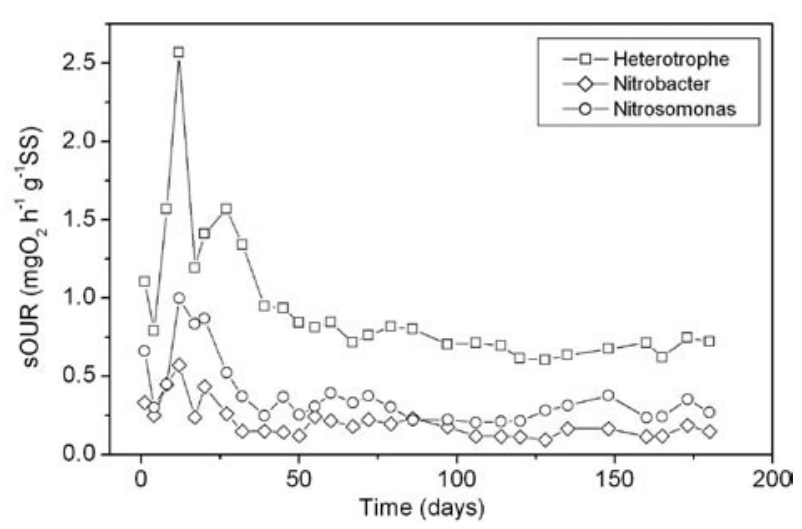

Figure 11

Variations of sOUR of different bacterial over the operation time

- The sludge activity was possibly inhibited by the accumulated SMP or affected by the pump shear stress. sOUR decreased with MLSS increasing in the reactor, which could be expressed by an exponential function. Despite maintaining the sOUR of the Nitrosomonas group and the Nitrobacter group at a low level, the nitrifying capacity of CDMBR remained high enough to qualify nitrogen removal.

\section{References}

ARNOT TC and ZAHIR N (1996) Membrane bioreactors as an alternative to conventional waste water treatment processes. Resour. Environ. Biotechnol. 1 145-162.

BROCKMA M and SEYFRIED CF (1996) Sludge activity and crossflow microfiltration-A non-beneficial relationship. Water Sci. Technol. 9 205-213.

CHINESE SEPA (1997) Water and Wastewater Monitoring Methods $\left(3^{\text {rd }}\right.$ edn.). Chinese Environmental Science Publishing House, Beijing.

CHOO KH and LEE CH (1996) Membrane fouling mechanisms in the membrane-coupled anaerobic bioreactor. Water Res. 30 1771-1780.

CHUDOBA J (1985) Inhibitory effect of refractory organic compounds produced by activated sludge micro-organisms on microbial activity and flocculation. Water Res. 19 197-200.

DUNCAN J and DAVID C (1999) A review of soluble microbial product (SMP) in wastewater treatment systems. Water Res. 33 3063-3082.

GANDER M, JEFFERSON B and JUDD S (2000) Aerobic MBRs for domestic wastewater treatment: a review with cost considerations. Sep. \& Pur. Technol. 18 119-130.
GORSKA JS, GERNAEY K, DEMUYNCY C, VANROLLEGHEM P and VERSTRAETE W (1996) Nitrification monitoring in activated sludge by oxygen uptake rate (OUR) measurements. Water Res. 30 1228-1236.

HALIL H, CUMALI K and AYHAN U (2002) Viability of microbial mass in submerged membrane bioreactor. Desalination 150263 268.

HUANG X, LIU R and QIAN Y (2000) Behavious of soluble microbial products in membrane bioreactor. Proc. Biochem. 36 401-406.

KIM JS, LEE, CH and CHANG IS (2001) Effect of pump shear on the performance of a crossflow membrane bioreactor. Water Res. 35 2137-2144.

LI F, TIAN Q, LI J, CHEN JH and DENG CH (2005) Performance of dynamic membrane in crossflow microfiltration: filtrating secondary effluent and activated sludge. Proc. Future of Urban Wastewater System-Decentralisation and Reuse. 493-502.

MARC A, RAPHASEL S and DAVID H (1999) Removal of organic foulants from feed waters by dynamic membranes. Desalination 125 65-75.

MEGAT MMN and FAKHRUL RA and THAMER AM (2002) Performance of flexible membrane using kaolin dynamic membrane in treating domestic wastewater. Desalination 147 263-268.

MUHAMMAD HA and ANDERSON GK (1997) Use of $\mathrm{MnO}_{2}$ as a dynamic membrane with crossflow microfiltration: slow membrane technique. Desalination 109 15-24.

NOWAK O and SVARDAL K (1997) Observation on the kinetics of nitrification under inhibiting conditions caused by industrial wastewater compound. WaterSci.. Technol. 28 115-123.

ROBERTS JA, PAUL MS and PRAKASH NM (2000) Application of the membrane biological reactor system for combined sanitary and industrial wastewater treatment. Intern. Biodeter. \& Biodegrad. 46 37-42.

RAPPAPORT SM, RICHARD MG, HOLLSTEIN MC and TALCOTT RE (1979) Mutagenic activity in organic wastewater concentration. Environ. Sci. Technol. 13 957-961.

ROSS N, DESCHENES L, BUREAU J and CLEMENT B (1998) Ecotoxicological assessment and effects of physicochemical factors on biofilm development in groundwater conditions. Environ. Sci. Technol. 32 1105-1111.

SHIN HS and KANG ST (2003) Characteristic and fates of soluble microbial products in ceramic membrane bioreactor at various sludge retention times. Water Res. 37 121-127.

RITTMANN BE, BAE W, NAMKUNG E and LU CJ (1987) A critical evaluation of microbial product formation in biological processes. Water Sci. Technol. 19 517-528.

WOUTER G, STEFAAN V and WILLY V (1999) Nitrogen removal from sludge reject water with a membrane-assisted bioreactor. Water Res. 33 23-32. 
Available on website http://www.wrc.org.za ISSN 0378-4738 = Water SA Vol. 32 No. 2 April 2006 ISSN 1816-7950 = Water SA (on-line) 